\title{
Analyzing Measles Vaccine Coverage in the United States
}

\author{
Daniel Goldman
}

January 2019

\section{Introduction}

Ever since Wakefield released his fraudulent paper, vaccine misinformation has been causing trouble for efforts to ensure significant vaccination coverage. The WHO even lists vaccine hesitancy as one of the top ten health concerns [5]. Measles cases in the United States are on the rise[4]. One of the primary causes, according to some of the medical community is the antivax movement[2]. However, insufficient analysis exists to make this claim. This short paper, which is part of the Improving Epidemiological Research project, looks at state and HHS region level data and seeks to identify whether coverage really has changed[3]. Answers to a number of questions were sought, in order to gain better insight into the matter.

1. Was there a downward change in the overall?

2. Was there a downward change in the minimum per state and per region coverage?

3. Was there an increase in the the variance of immunization rates between states and between regions?

\section{Method}

MMR vaccination coverage spanning the years 1995 - 2017, separated by state and HHS region, among children 19-35 months old was obtained from the CDC[1]. Data from Washington DC was also included. First, nation wide vaccination rates were investigated. Next, minimum vaccination rates, as well as standard deviation for each year were calculated, both by state and by region. While more complicated statistical methods are available, this paper is just an initial step to suggest the need for additional research, so linear regression was used. The slope, coefficient of determination, and $95 \%$ confidence intervals for slopes of each category were calculated. 


\section{Results}

Table 1

\begin{tabular}{|c|c|c|c|c|}
\hline Statistic & Slope & LB & UP & Adjusted $R^{2}$ \\
\hline \hline Nation Wide & 0.04348 & -0.01377205 & 0.1007286 & 0.0636 \\
\hline Region Minima & 0.04931 & -0.0173152 & 0.1159318 & 0.05858 \\
\hline State Minima & 0.08320 & -0.01513057 & 0.1815337 & 0.087 \\
\hline Region STD & -0.014182 & -0.03339288 & 0.005028885 & 0.0581 \\
\hline State STD & -0.01952 & -0.04541078 & 0.006369582 & 0.06218 \\
\hline
\end{tabular}

Table 1: Regression Results

\section{Discussion}

There were a number of limitations with this analysis. First, the data only considered those who had received at least one dose. However, because the overall claim being tested was that vaccine hesitancy is resulting in an increase in cases, it would be somewhat unusual for these results to hold. It does not make sense that a vaccine hesitant parent would accept one dose but not additional doses. Another limitation is that the highest resolution of data was state level data. County data might provide additional information.

However, based on the data analysis conducted so far, there is reason to be concerned about premature assignment of blame. We are seeing a positive trend in minimum vaccination rates, as well as a smoothing of vaccination coverage. While county wide pockets may very well be forming, coverage at the state and regional levels are becoming increasingly even. This result is somewhat puzzling, considering the increasing sentiment regarding vaccine hesitancy. One possibility is that this analysis is simply not properly capturing the impact on vaccination rates. In all four analyses, the $95 \%$ confidence interval for the slope passed through 0. Another option is that while people may be becoming increasingly vocal, and in some small areas may be refusing to vaccinate, the rhetoric may not be translating into action.

In order to get a better picture, county level data is going to be needed. It may also be useful to perform a non-linear analysis, and possibly a graphical analysis on the data. The goal would be to look for the emergence of clusters that could be acting as a source of infection for the general population.

\section{References}

[1] ChildVaxView - 2013-2017 Childhood MMR Vaccination Coverage Trend Report - CDC. URL: https://www.cdc.gov/vaccines/imz-managers/ coverage/childvaxview/data-reports/mmr/trend/index.html (visited on $01 / 27 / 2019$ ). 
[2] Azhar Hussain et al. "The Anti-vaccination Movement: A Regression in Modern Medicine". In: Cureus (July 2018). DOI: 10.7759/cureus. 2919. URL: https://doi.org/10.7759/cureus . 2919.

[3] Improving Epidemiological Research - Daniel Goldman - 1 updates Research Project. URL: https : / / www . researchgate . net / project / Improving-Epidemiological-Research (visited on 01/27/2019).

[4] Measles - Cases and Outbreaks - CDC. URL: https://www.cdc.gov/ measles/cases-outbreaks.html (visited on 01/27/2019).

[5] Ten threats to global health in 2019. URL: https://www . who. int/emergencies/ ten-threats-to-global-health-in-2019 (visited on 01/27/2019). 